\title{
Selective Depletion of Chiral 4-Hydroxypraziquantel Metabolites in Three Types of Aquaculture Fish by LC-MS/MS
}

\author{
Yingxia Zhang, ${ }^{a,+}$ Limin He ${ }^{a, b,+, *}$, Xuezhi Li ${ }^{a}$, Yadi Wang ${ }^{b}$, Jingmeng Xia ${ }^{a}$, Jauh Tzuoh Lee ${ }^{c}$ and Daniel \\ W. Armstrong ${ }^{\mathbf{b}, \mathbf{c}, *}$
}

a National Reference Laboratory of Veterinary Drug Residues (SCAU), College of Veterinary Medicine, South China Agricultural University, Guangzhou 510642, China

${ }^{b}$ Department of Chemistry and Biochemistry, The University of Texas at Arlington, Arlington, TX 76019, USA

${ }^{c}$ AZYP LLC, Arlington, TX 76019, USA

+ The authors contributed equally to this work.

* Correspondence: Dr. Limin He, National Reference Laboratory of Veterinary Drug Residues (SCAU), College of Veterinary Medicine, South China Agricultural University, Guangzhou, 510642, China. Tel. and Fax: +(086)-20 85284896.

E-mail: liminokhe@scau.edu.cn

* Corresponding author at: Department of Chemistry and Biochemistry, The University of Texas at Arlington, Arlington, TX 76019, USA. Tel.: +1 817272 0632; fax: +1 8172720619 .

E-mail address: sec4dwa@uta.edu (D.W. Armstrong) 

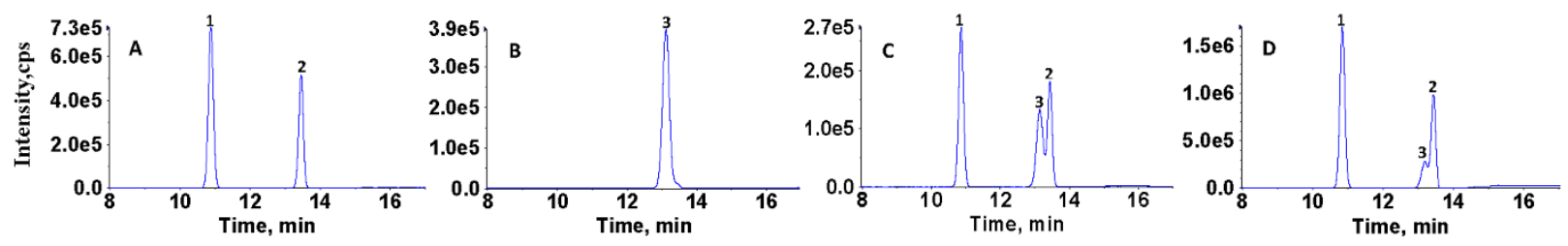

Figure S1. SRM chromatograms of 4-OH-PZQ (A), 3-OH-PZQ (B), 4-OH-PZQ+3-OH-PZQ (C), perch muscle sample after administration (D) separated by $\mathrm{C}_{18}$ column . Chromatographic conditions: mobile phase, 0 1 min, 5\% acetonitrile in $10 \mathrm{mM}$ ammonium formate buffer solution ( $\mathrm{pH} 3.6$ ); $1 \sim 3 \mathrm{~min}, 5 \% \sim 25 \%$ acetonitrile; $3 \sim 10 \mathrm{~min}$, keep 25\% acetonitrile; 10 12 $\mathrm{min}, 25 \% \sim 48 \%$ acetonitrile; $12 \sim 18 \mathrm{~min}$, keep $48 \%$ acetonitrile; $18.1 \mathrm{~min}, 5 \%$ acetonitrile; flow rate, $0.2 \mathrm{~mL} \mathrm{~min}^{-1}$; the column temperature, $25^{\circ} \mathrm{C}$ (Signal channel: $m / z 329.3 / 311.3)$.

1, 2 and 3, represent trans-4-OH-PZQ, cis-4-OH-PZQ and R + S-3-OH-PZQ, respectively. 


\section{Table S1}

Linear range and regression equations for 4-hydroxypraziquantel $(n=6)$.

\begin{tabular}{|c|c|c|c|c|}
\hline Fish muscle & $\begin{array}{l}\text { Concentration range } \\
\left(\mu \mathrm{g} \mathrm{L}^{-1}\right)\end{array}$ & Compound & regression equation & $\overline{r^{2}}$ \\
\hline \multirow[t]{4}{*}{ perch } & $1-250$ & R-trans-4-OH-PZQ & $y=5.67 \times 10^{5} x+1.36 \times 10^{4}$ & 0.9993 \\
\hline & & S-trans-4-OH-PZQ & $y=5.52 \times 10^{5} x+2.23 \times 10^{4}$ & 0.9991 \\
\hline & & R-cis-4-OH-PZQ & $y=4.31 \times 10^{5} x+3.48 \times 10^{4}$ & 0.9999 \\
\hline & & S-cis-4-OH-PZQ & $y=4.36 \times 10^{5} x+2.28 \times 10^{4}$ & 0.9996 \\
\hline \multirow[t]{4}{*}{ tilapia } & $1-250$ & R-trans-4-OH-PZQ & $y=4.09 \times 10^{5} x+1.37 \times 10^{4}$ & 0.9990 \\
\hline & & S-trans-4-OH-PZQ & $y=4.05 \times 10^{5} x+1.46 \times 10^{4}$ & 0.9998 \\
\hline & & R-cis-4-OH-PZQ & $y=3.48 \times 10^{5} x+1.27 \times 10^{4}$ & 0.9996 \\
\hline & & S-cis-4-OH-PZQ & $y=3.37 \times 10^{5} x+1.13 \times 10^{4}$ & 0.9993 \\
\hline \multirow[t]{4}{*}{ ricefield eel } & $1-250$ & R-trans-4-OH-PZQ & $y=5.07 \times 10^{5} x+2.01 \times 10^{4}$ & 0.9999 \\
\hline & & S-trans-4-OH-PZQ & $y=5.18 \times 10^{5} x+1.39 \times 10^{4}$ & 0.9997 \\
\hline & & R-cis-4-OH-PZQ & $y=4.38 \times 10^{5} x+1.01 \times 10^{4}$ & 0.9991 \\
\hline & & S-cis-4-OH-PZQ & $y=4.27 \times 10^{5} x+1.18 \times 10^{4}$ & 0.9996 \\
\hline
\end{tabular}

